\title{
Dependence of developing magnetic hysteresis characteristics on stages of evolving microstructure in polycrystalline yttrium iron garnet.
}

\begin{abstract}
The microstructure evolution in several polycrystalline yttrium iron garnet samples as a result of a sintering scheme was studied in detail, in parallel with the changes in their magnetic properties. Samples with nanometer sized starting powder were synthesized by employing the High-Energy Ball Milling technique and then sintering toroidal compacts of the milled powder. Nine sintered samples were obtained, each corresponding to a particular sintering from $600{ }^{\circ} \mathrm{C}$ to $1400{ }^{\circ} \mathrm{C}$. The samples were characterized for their evolution in crystalline phases, microstructure and magnetic hysteresis-loops parameters. The results showed an increasing tendency of the saturation magnetization and saturation induction with grain size, which is attributed to crystallinity increase and to reduction of demagnetizing fields in the grains. The variation in coercivity could be related to anisotropy field changes within the samples due to grain size changes. In particular, the starting appearance of room temperature ferromagnetic order suggested by the sigmoid-shaped B-H loops seems to be dependent on a sufficient number of large enough magnetic domain-containing grains having been formed in the microstructure. Viewed simultaneously, the hysteresis loops appear to belong to three groups with different magnetism-type dominance, respectively dependent on phase purity and three different groups of grain size distributions.
\end{abstract}

Keyword: Sintering temperature; BH hysteresis behaviour; Magnetic properties; Microstructure; Sintering temperature. 